\title{
Energy Efficient Linear and Non-Linear Precoders for Massive MIMO Systems
}

\author{
Samson Hansen Sackey ${ }^{1}$, Michael Kwame Ansong², Samuel Nartey Kofie ${ }^{3}$, Abdul Karim Armahy \\ 1, 2, 3 College of Internet of Things, Hohai University, Changzhou, 213022, China. \\ ${ }^{4}$ School of Managerial Engineering, Zhengzhou University, Zhengzhou, 450001, China.

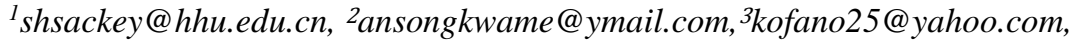 \\ 4yarmahabdulkarim1@yahoo.com
}

\begin{abstract}
The term Massive MIMO means, Massive multiple input multiple output also known as (large-scale antenna system, very large MIMO). Massive Multiple-Input-MultipleOutput (MIMO) is the major key technique for the future Fifth Generation $(5 \mathrm{G})$ of mobile wireless communication network due to its characteristics, elements and advantages. Massive MIMO will be comprised of five major elements; antennas, electronic components, network architectures, protocols and signal processing. We realize that precoding technique is a processing technique that utilizes Channel State Information Technique (CSIT) by operating on the signals before transmitting them. This technique varies base on the type of CSIT and performance criterion. Precoding technique is the last digital processing block at the transmitting side. In this paper, linear and non-linear Precoding technique was reviewed and we proposed two techniques under each that is Minimum Mean Square Error (MMSE), Block Diagonalization (BD), Tomlinson-Harashima (TH) and Dirty paper coding (DPC). Four Precoding techniques: MMSE, BD, DPC and TH were used in the studies to power consumption, energy efficiency and area throughput for single-cell and multi-cell scenarios. In comparing the proposed techniques, in terms of energy efficiency and area throughput, reuse factor (Reuse 4) performs better than other techniques when there is an imperfect CSI is used.
\end{abstract}

Keywords: Massive MIMO, Precoding, CSI, antennas, base station, signals, linear/non-linear precoders.

\section{INTRODUCTION}

Wireless communication was introduced in the 19th century and wireless communication technology has developed over the last decade and it one of the most important mediums of transmission of information from one device to other. The term wireless is often used to describe all types of devices and technologies not connected by a wire. A garage door opener and a television remote control are some examples of wireless devices. Devices like notebook computers, tablets, digital still picture and video cameras, printers, portable digital music players, even refrigerators, washing and drying machines, and electricity meters are equipped with the ability to communicate without wires [1] [2].

Wireless communications are defined as the transmission of digital data without the use of wires, meaning devices that can be interconnected using some kind of data networking technology. Digital data may include e-mail messages, spreadsheets, and messages transmitted to or from a digital cellular phone.

It can also be defined as, transmitting of information through the air without requiring any cable or wires or other electronic conductors, by using electromagnetic waves like IR (Infrared), RF, satellite, etc. [3]. Wireless communications affect almost everything we do on a daily basis, like using cellular phones

to making voice calls and accessing information, counting of inventory in large retail stores, buying public transit system tickets, locating hotspots for Internet access and wireless remote sensors installed in locations that are difficult to access, using credit and debit cards that just need to be placed near a device instead of swiped or inserted, etc. There should be no question in anyone's mind 
that the use of wireless devices will continue to expand into virtually every aspect of our lives [4]. Wireless communication has the following advantages: To enhance information quickly to the consumers. Working professionals can work and access the Internet anywhere and anytime without carrying cables or wires wherever they go.

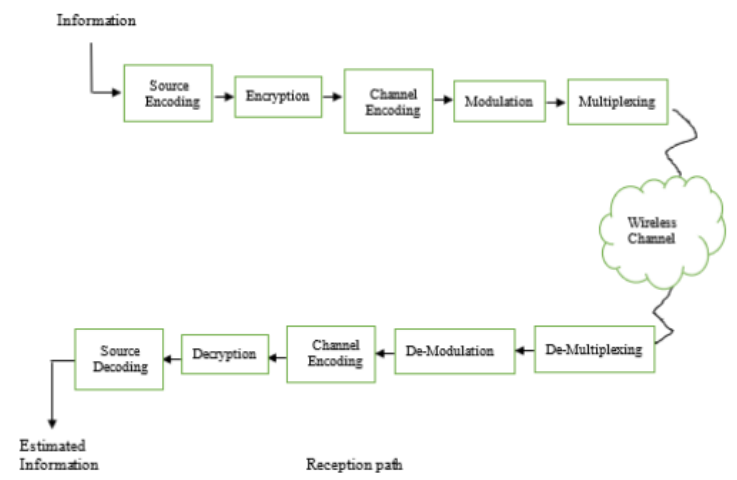

Fig. 1. Block Diagram of Wireless Communications System

This also helps to complete the work anywhere on time and improves the productivity [5]. Doctors, workers and other professionals working in remote areas can be in touch with medical centers through wireless communication. An urgent situation can be alerted through wireless communication. The affected regions can be provided help and support with the help of these alerts through wireless communication. Wireless networks are cheaper to install and maintain. We can be in touch with the digital resources we need, no matter where we may find ourselves [6]. Nearly everyone has experienced dramatic changes based on wireless technologies, to the extent that we dont even think about what we are doing, we just expect devices [7]. The key contributions made in this paper are next:

- Four Precoding techniques namely MMSE, $\mathrm{BD}, \mathrm{DPC}$ and TH were used in the studies in terms of power consumption, energy efficiency and area throughput for single-cell and multi-cell scenarios.

- In comparing the proposed techniques, in terms of energy efficiency and area throughput, reuse factor (Reuse 4) performs better than other techniques when there is an imperfect CSI is used

The rest of the paper is structured as follows. Review of related work is described in Section II. The system model and the proposed approach for precoders MIMO systems is declared in Section III. Performance analysis with some existing conventional approach is described in Section IV. To end with, we conclude in section $\mathrm{V}$.

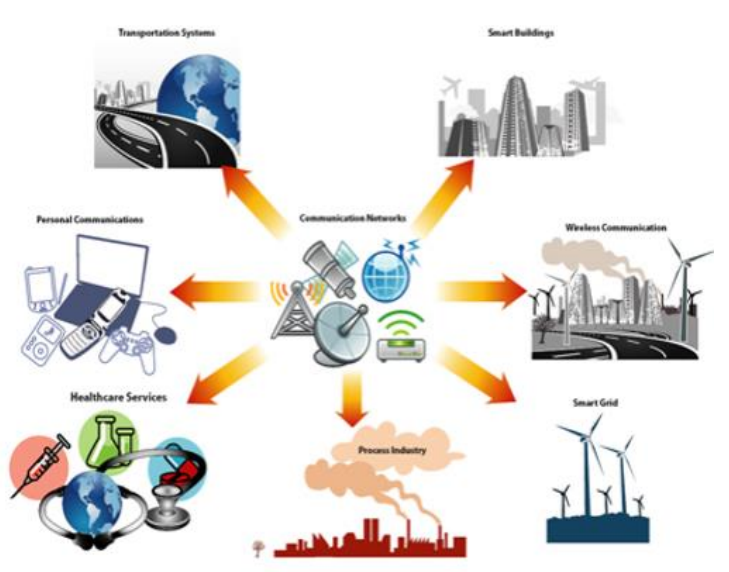

Fig. 2. Wireless Communications Application

\section{RELATED WORKS}

MIMO (multiple input multiple output) is a method of increasing the performance of radio link using multiple transmit and receive antennas to achieve a multipath propagation [8]. It can also be the use of multiple antennas at the transmitter and the receiver or technique for sending and receiving more than one data signal simultaneously on the same radio channel by performing multipath propagation [9],[14]. It is an important element of wireless communication standards which includes IEEE 802 [10].11n (Wi-Fi), IEEE 802.11ac (WiFi), HSPA+ (3G), WiMAX (4G) and Long-Term Evolution (4G).

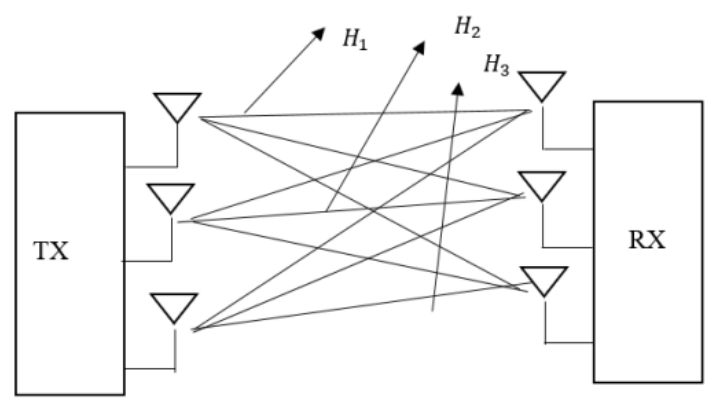

Fig. 3. Block diagram of MIMO Systems

Massive MIMO is a Multi-user MIMO (MuMIMO) with a large number of Base Station (BS) antennas and users and it has less number of transmitter terminals than the BS antennas [11]. This system uses antenna arrays with a very large number of antennas elements that makes use of service antennas over active terminal and Time Division Duplex (TDD) operation on a large scale [12],[23]. This technology is an upgrade of the current MIMO (Mu-MIMO) and extract all the 
S. H. Sackey et. al / International Journal of Computer Networks and Communications Security, 8 (8), August 2020

benefits of it but on a large scale. In this system, hundreds or thousands of BS antennas serves tens or hundreds of users simultaneously in the same time-frequency channel [13]. Massive MIMO is the next Generation network that is expected to be lunch across the globe by the year 2020 [15]. The concept of Mu-MIMO

System was proposed in 1991 by Richard Roy and Bjorn Ottersten [23]. Massive MIMO system components will have different ways of accessing higher frequency ranges and the motive of massive antenna setups are going to be direct-to device communication and ultra-dense deployments [24].

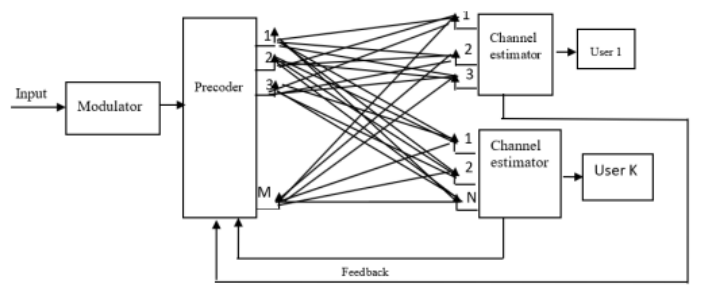

Fig. 4. Block diagram of MIMO Systems

Signal processing deals with the impairments of the physical medium and acquiring a low-cost effective tool for processing information is an extremely role for MIMO signal processing [16]. This signal processing needs a computational cost for transmitting and receive processing which grows as cubic or super-cubic functions of the number of antennas that is certainly not effective with a large number of antennas elements [17]. The need of simpler solutions for the transmit and receive processing task will need signal processing under Massive MIMO System will require novel signal processing to deal with all the problems like computational complexity and its scalability, RF impairment, coupling effects, pilot contamination, delay and calibration issues [18] [19].

This is a processing technique that utilizes CSIT by operating on the signals before transmitting them [20], [21]. This technique varies base on the type of CSIT and performance criterion [22] and it is the last digital processing block at the transmitting side as shown in Figure 7. Improvement of link performance and reduction of the corrupting effect of the communication channel are some of the main aims of this technique. In precoding technique, every transmits signals processing involves CSIT [25].

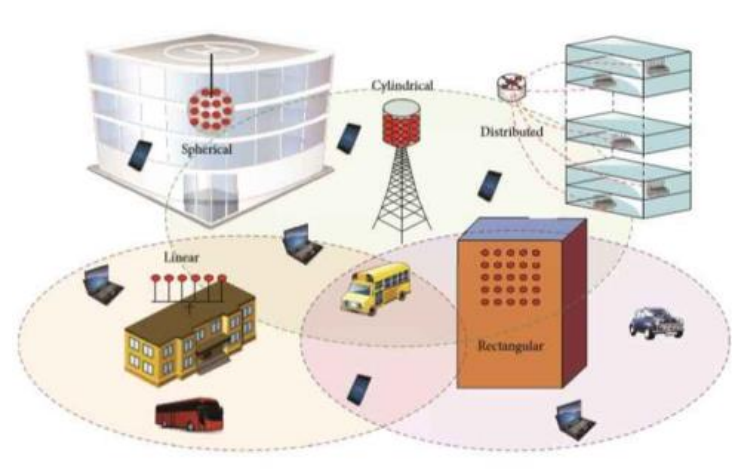

Fig. 5: Massive MIMO System Antenna Configurations

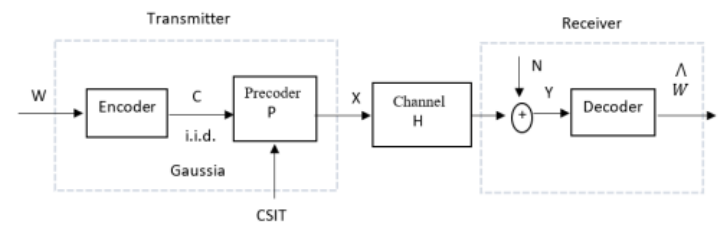

Fig. 6. The structure of Precoding System

\section{APPROACH TO LINEAR AND NON- LINEAR PRECODING TECHNIQUES}

\section{A. System Model}

Consider the Massive MIMO broadcast channel with, $N_{t}$ transmit antennas at the base station (BS), $N_{i}$ receive antennas at $i^{t} h$ user equipment, $K$ number of users equipment and $N$ number of receive antennas per user. The total number of receive antennas can be represented as [23]:

$$
N_{r}=\sum_{i=1}^{k} N_{i}
$$

The received signal is represented by $\mathrm{y}$, for the $i^{t} h$ user equipment, the received signal is represented as:

$$
y_{i}=H_{i} x+n_{i}
$$

Which is also represented by independent and identical distribution matrix:

$$
\left[\begin{array}{c}
y_{1} \\
\vdots \\
y_{i}
\end{array}\right]=\left[\begin{array}{ccc}
H_{1} & 0 & 0 \\
\vdots & \vdots & \vdots \\
0 & 0 & H_{i}
\end{array}\right]\left[\begin{array}{c}
x_{1} \\
\vdots \\
x_{i}
\end{array}\right]+\left[\begin{array}{c}
n_{1} \\
\vdots \\
n_{i}
\end{array}\right]
$$


S. H. Sackey et. al / International Journal of Computer Networks and Communications Security, 8 (8), August 2020

Where $H_{i}$ is the $i t h$ users channel gain matrix, $x$ is the transmitted symbol of the user and $n_{i} C$ $\left(N_{r} 1\right)$ represents the $i t h$ users AWGN noise symbol vector of the user $i$ having the size of $\left(N_{t} 1\right)$ for the information transmitted signals. $P$ precoding matrix. At the base station, the perfect Channel State Information (CSI) receive as a channel gain matrix $H$. Whiles imperfect CSI is given by a noisy channel matrix.

$$
\begin{gathered}
H_{e}=H+E \\
H=\left[H_{1}^{T} \ldots H_{k}^{T}\right]^{T} \in C^{N_{r} \times N_{t}} \\
P=\left[P_{1} \ldots P_{k}\right]^{T} \in C^{N_{r} \times N_{t}}
\end{gathered}
$$

Where $H(i) C\left(N_{i} N_{t}\right)$ is the $i t h$ users channel matrix. The quantity $P(i) C_{(}\left(N_{t} N_{i}\right)$ is the $i t h$ users precoding matrix.

\section{B. Linear Precoders Strategy}

In this section, the minimum square error (MMSE) and block diagonalizaton algorithm procedure is described briefly as follows. In linear precoding, we employed the technique of MMSE to reduce throughput to avoid constantly presence of zero-forcing precoding. To minimize the presences of MSE (mean squared error), the precoding is given by:

$$
\min _{p} \sum_{i=1}^{k}\left|x_{i}-y_{i} / E^{(i, i)}\right|^{2}
$$

Where $\left.E_{(}(i ; i)\right)$ signifies the $i t h$ diagonal element of the matrix. Again, the precoding matrix of MMSE is given as:

$$
P_{i}=H_{i}^{H}\left(H_{i} H_{i}^{H}+\beta I_{i}\right)^{-1}
$$

Where $P_{i}$ indicates the precoding matrix for the user $i t h$ with size of $(N(i) N)$, I is the identity matrix and $i$ represents the normalization factor, which is given as:

$$
\beta_{i}=\frac{N_{t}}{S N R_{i}}
$$

We discuss the Block Diagonalization (BD) algorithm and the procedure to obtain the precoding matrix $\mathrm{P}$. In $\mathrm{BD}$ algorithms, the first precoding filter is used to transform a Massive MIMO channel into a block channel. The reduced channel matrix for the ith user is defined as

$$
\overline{H_{i}}=\left[H_{1}^{T} \ldots H_{i+1}^{T} \ldots H_{K}^{T}\right]^{T} \in C^{\overline{N_{i}} \times \overline{N_{t}}}
$$

Where $N_{i}=N_{r}-N_{i}$. The first precoding matrix is given as:

$$
P_{i}^{\alpha}=\bar{V}_{i}^{(o)}
$$

Where, $\left.V \quad{ }_{(i}(0)\right)\left(N_{t}\left(N_{t}-R_{i}\right)\right)$ is obtained by performing the SVD decomposition of $H_{i} . R_{i}$ is the rank of the channel gain matrix $H_{i}$. The combined precoding matrix is given by,

$$
P_{i}=P_{i}^{\alpha} P_{i}^{\beta}
$$

Where, $P_{i a}\left(N_{t} L_{i}\right)$ and $P_{i b}\left(L_{i} N_{i}\right)$. The parameter $L_{i}$ is

dependent on the specific choice of the precoding algorithm.

\section{Non-Linear Precoders Approach}

Algorithms for Tomlinson-Harashima and Dirty Paper Coding of non-linear Precoding are as follows with the same system model as the linear Precoding. Where $F$ is the $N_{t} K N$ feedforward matrix obtained by an $L Q$ decomposition of the channel matrix $H$ and the input is computed element by the element. Where biqare the elements of the $K N K N$ lower triangular matrix.

$$
\begin{gathered}
x[i]=F \tilde{x}[i] \\
\tilde{x}[i]=\bmod \left\{s_{l}[i]-\sum_{q=1}^{l-1} b_{l q} x_{q}[i]\right\}, l=1
\end{gathered}
$$

This technique of interference transmission can be known by subtracting the potential interference before transmission.

It is very important to know the channel gain completing at the transmitter side before implementing DPC. These Precoding techniques are implemented when the channel gains are completely known on the transmitter side. If $\mathrm{K}=3$ user, the received signal is given as:

$$
\left[\begin{array}{l}
y_{1} \\
y_{2} \\
y_{3}
\end{array}\right]=\left[\begin{array}{l}
H_{1}^{D L} \\
H_{2}^{D L} \\
H_{3}^{D L}
\end{array}\right]\left[\begin{array}{l}
X_{1} \\
X_{2} \\
X_{3}
\end{array}\right]+\left[\begin{array}{c}
Z_{1} \\
Z_{2} \\
Z_{3}
\end{array}\right]+\left[\begin{array}{c}
n_{1} \\
n_{2} \\
n_{3}
\end{array}\right]
$$

The channel matrix $H_{D} L$ can be $L Q$ decomposed as: 


$$
H^{D L}=\left[\begin{array}{ccc}
l_{11} & 0 & 0 \\
l_{21} & l_{22} & 0 \\
l_{31} & l_{32} & l_{33}
\end{array}\right]\left[\begin{array}{l}
q_{1} \\
q_{2} \\
q_{3}
\end{array}\right]
$$

$H_{D}$ i $L C(13)$ is the channel matrix between BS and $i t h$ user. The received signal for the first user is given as:

$$
\begin{gathered}
y_{1}=l_{11} x_{1}+z_{1}+n_{1} \\
x_{1}=\overline{X_{1}}
\end{gathered}
$$

Received signal for $K=3$ user,

$$
y_{3}=l_{31} x_{1}+l_{32} x_{2}+l_{33} x_{3}+z_{3}+n_{3}
$$

Combining equations, $P$ is the precoding signals expression:

$$
\left[\begin{array}{l}
x_{1} \\
x_{2} \\
x_{3}
\end{array}\right]=P\left[\begin{array}{c}
\tilde{x}_{1} \\
\widetilde{x_{2}} \\
\widetilde{x_{3}}
\end{array}\right]
$$

The equation can be re-write as;

$$
\left[\begin{array}{l}
y_{1} \\
y_{2} \\
y_{3}
\end{array}\right]=\left[\begin{array}{ccc}
l_{11} & 0 & 0 \\
0 & l_{22} & 0 \\
0 & 0 & l_{33}
\end{array}\right]\left[\begin{array}{l}
\overline{x_{1}} \\
x_{2} \\
x_{3}
\end{array}\right]+\left[\begin{array}{l}
z_{1} \\
z_{2} \\
z_{3}
\end{array}\right]
$$

Base on the above formulations, the channel gain matrix can be obtained by considering the scaled inverse matrix of the lower triangular matrix.

$$
P=\left[\begin{array}{ccc}
l_{11} & 0 & 0 \\
l_{21} & l_{22} & 0 \\
l_{31} & l_{32} & l_{33}
\end{array}\right]^{-1}\left[\begin{array}{ccc}
l_{11} & 0 & 0 \\
0 & l_{22} & 0 \\
0 & 0 & l_{33}
\end{array}\right]
$$

\section{SIMULATION RESULT AND PERFORMANCE ANALYSIS}

Simulation of energy efficiency, average power, radio frequency power and radiated power per BS for number of antennas and the number of users were conducted together with all the proposed Precoding techniques that are MMSE, BD, DPC and TH. MATLAB was the software for the simulation. Figure 7 , shows the flowchart of the simulation process.

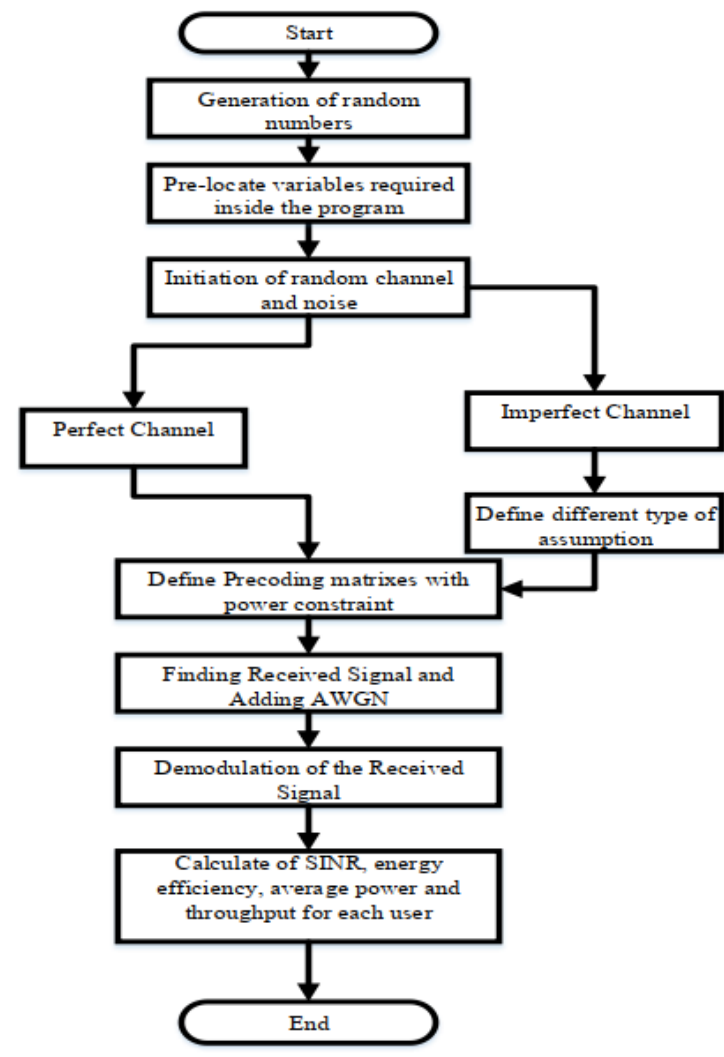

Fig. 7. A Flow Chart of the Simulation Process.

We have demonstrated in Figure 8.0 the probably achieved energy efficiency values with proposed techniques in perfect CSI for diverse $K$ and $N_{t}$ values. This provides an optimal energy efficiency that is resilient to rapid changes in distributed network system.

Table 1: Simulation Parameters

\begin{tabular}{|l|l|}
\hline Parameter used & Value \\
\hline No. of base station antenna(BS) & 200 \\
\hline Energy efficiency & 35 \\
\hline No. of active users & 150 \\
\hline Cell radius: $d$ max & $250 m$ \\
\hline $\begin{array}{l}\text { Distance between Users and base sta- } \\
\text { tion }\end{array}$ & $35 m$ \\
\hline Average power & $10^{-2}-10^{2}$ \\
\hline Area throughput & $0-9$ \\
\hline Coverage area (area Single cell) & $p i$ \\
$(d \mathrm{max} / 1000)^{2}$ \\
\hline Coverage area (area multi cell) & $4 *(d \mathrm{max} / 1000)$ \\
\hline $\begin{array}{l}\text { Regulate channel attenuation at mini- } \\
\text { mum distance }\end{array}$ & $10^{-3.53}$ \\
\hline Path- loss exponent & 3.76 \\
\hline Transmission bandwidth & $20 \mathrm{MHz}$ \\
\hline
\end{tabular}




\begin{tabular}{|l|l|}
\hline Channel coherence bandwidth & $180 \mathrm{kHz}$ \\
\hline Channel coherence time & $10 \mathrm{~ms}$ \\
\hline Total noise power & $-96 \mathrm{dBm}$ \\
\hline Fraction of downlink transmission & 0.4 \\
\hline Fraction of uplink transmission & 0.5 \\
\hline $\begin{array}{l}\text { Power required to run the circuit com- } \\
\text { ponents at BS }\end{array}$ & $1 \mathrm{~W}$ \\
\hline $\begin{array}{l}\text { Power required to run the circuit com- } \\
\text { ponents at Users }\end{array}$ & $0.1 \mathrm{~W}$ \\
\hline $\begin{array}{l}\text { Power required for coding of data } \\
\text { signal signals }\end{array}$ & $0.1 \mathrm{~W} /(\mathrm{Gbit} / \mathrm{s})$ \\
\hline $\begin{array}{l}\text { Power required for decoding of data } \\
\text { signal signals }\end{array}$ & $0.8 \mathrm{~W} /(\mathrm{Gbit} / \mathrm{s})$ \\
\hline
\end{tabular}

Simulation was conducted for MMSE and BD for energy efficiency and average power and below are the results: Simulation was conducted for $\mathrm{TH}$ and DPC for area throughput and below is the result. From figure 9 and 10, linear precoding method was used to verify the appropriate techniques. Similarly, Figure 11 as nonlinear precoding techniques, verified the same approachable techniques. For the cases of multi-cell scenarios in Figures 9 to 11, similar conclusions are achieved as in single-cell scenarios using few antennas. Therefore, there is reduction in transmit power consumption and area throughput which affects the energy efficiency in the network. We observed that the highest reuse factors (Reuse 4) yields better area throughput and maximum energy efficiency.

\section{CONCLUSION AND FUTURE WORK}

Massive MIMO using Precoding techniques have proved to improve signal transmission from the transmitter through the cooperative relays to the receiver. Multiple antennas are used at both at both transmit and receive side. Four Precoding

techniques: MMSE, BD, DPC and TH were used in the studies to power consumption, energy efficiency and area throughput for single-cell and multi-cell scenarios. In comparing the proposed techniques in terms of energy efficiency and area throughput in reuse factor (Reuse 4) performs better than other techniques when there is an imperfect CSI is used. We also realize that energy efficiency increases as the number of users are increasing, more antennas are needed for signal transmission from sorts to destination and when the number of users' increases with less number of antennas there will be more power and energy consumption. In future, we hope to apply the precoding techniques based on reuse perfect CSI in signal transmission.

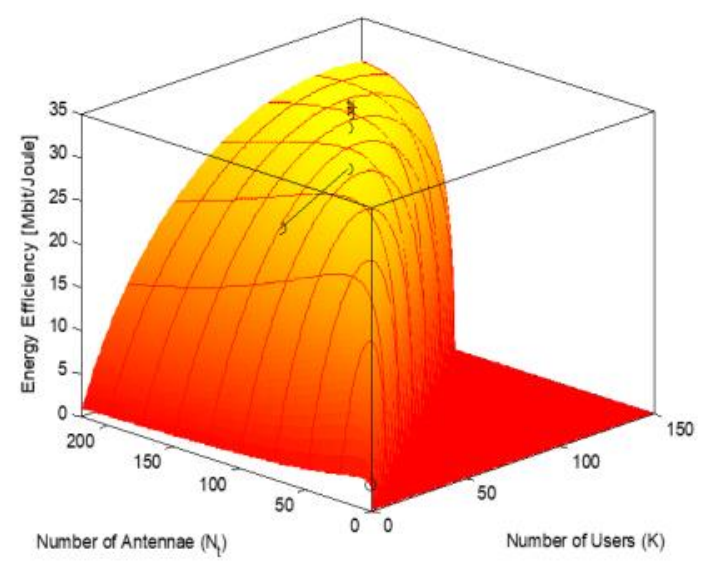

Fig. 8. A plot of energy efficiency with precoding techniques in the single-cell

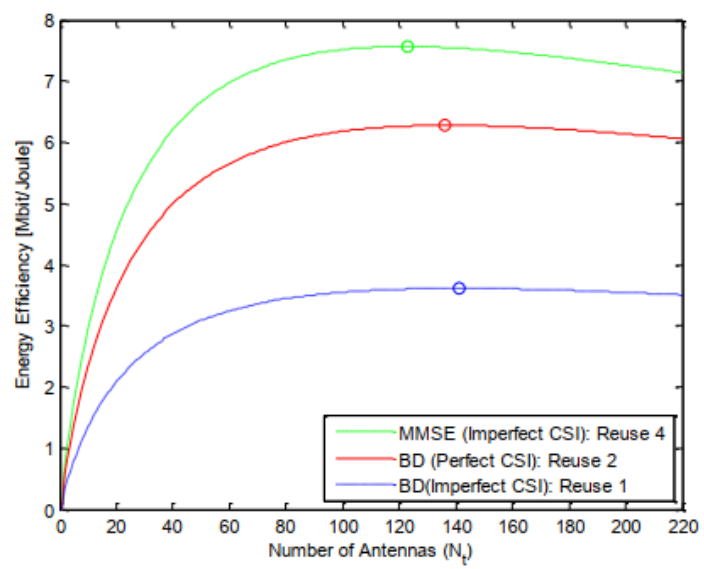

Fig. 9. A plot of energy efficiency in the multi-cell scenario for different number of precoding antennas and different pilot factors. 
S. H. Sackey et. al / International Journal of Computer Networks and Communications Security, 8 (8), August 2020

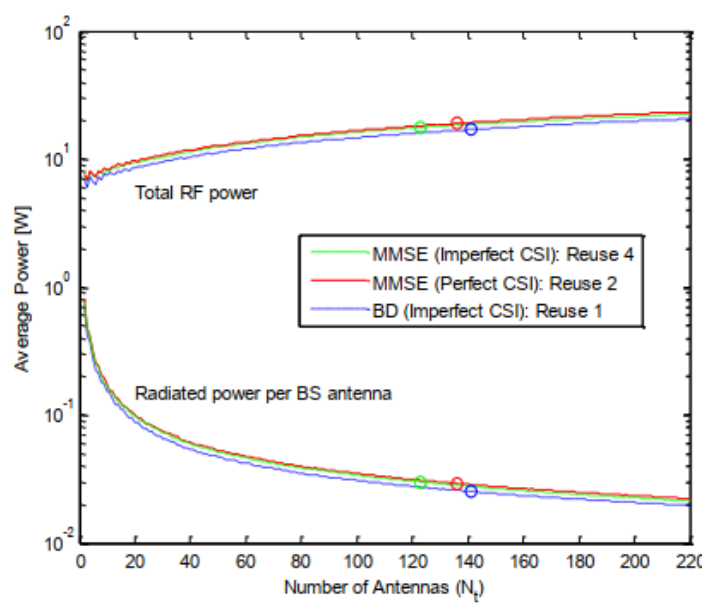

Fig. 10. A plot of average power in maximizing energy efficiency for multi-cell scenario, using different precoding antennas different reuse factors.

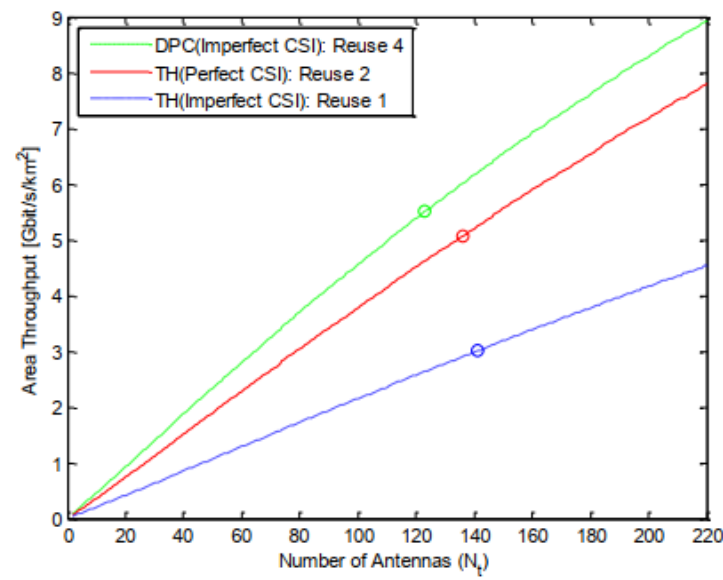

Fig. 11. A plot of area throughput in maximizing energy efficiency for multi-cell.

\section{REFERENCES}

[1] Jorge L. Olenewa,"Guide to Wireless Communications Third Edition", Course Technology, pp.1-20, 2012.

[2] S. H. Sackey, G. K. Gapko, S. N. Kofie, and A. K. Armah, "Inspiring Evolving Technologies in Internet of Things", Pacific International Journal, vol. 2, no. 2, pp. 41-47, 2019.

[3] P. Sharma, "Evolution of Mobile Wireless Communication Networks-1G to $5 \mathrm{G}$ as well as Future Prospective of Next Generation Communication Network", Computer Science and Information Technology, vol. 2, pp. 47-53, 2013.

[4] J. Hoydis, M. Kobayashi, and M. Debbah, "Green small-cell networks", IEEE Vehicular Technology Magazine, pp. 3743, March, 2011.

[5] H. Yanikomeroglu, "The building blocks of the 5 th generation $(5 \mathrm{G})$ wireless networks for affordable and ubiquitous", Tech. Rep., BCWS Seminar Series, Carleton University, 2011.

[6] Shahid Mumtaz, Jonathan Rodriguez and Linglong Dai,"mmWave Massive MIMO a Paradigm for 5G", Academic Press, 2017.

[7] J. A. Ansere, J. H. Anajemba, S. H. Sackey, C. Iwendi and M. Kamal,"Optimal Power Distribution Algorithm for Energy Efficient IoT-NOMA Enabled Networks," 2019 15th International Conference on Emerging Technologies (ICET), Peshawar, Pakistan, 2019, pp. 1-5, doi: 10.1109/ICET48972.2019.8994373.

[8] B. Lars T., S. Andreas, P. Pascal, S. Daniel M.,"MIMO Power Line Communications: Narrow and Broadband Standards, EMC and Advanced Processing", Devices, Circuits and Systems, February, 2014.

[9] S. H. Sackey, J. Chen, S. N. Kofie, and N. Bulgan, "Brain Storm Optimization for Energy-Saving Routing Algorithm in Wireless Sensor Networks", International Journal of Scientific and Research Publica- tions (IJSRP), vol. 9, issue 5, 2019.

[10] J. H. Anajemba, Y. Tang, J. A. Ansere and S. H. Sackey, "Efficient Switched Digital Beamforming Radar System based on SIMO/MIMO Receiver,” 2019 Computing, Communications and IoT Applications (ComComAp), Shenzhen, China, 2019, pp. 411-416, doi: 10.1109/ComComAp46287.2019.9018789.

[11]F. Rusek et al.,"Scaling Up MIMO: Opportunities and Challenges with Very Large Arrays," IEEE Sig. Proc. Mag., vol. 30, Jan., pp. 4060, 2013.

[12]E. G. Larsson, ISY, Ove Edfors, F. Tufvesson, T. L. Marzetta, B. Labs and AlcatelLucent,"Massive MIMO for Next Generation Wireless Systems", IEEE Communications Magazine, February, 2014.

[13] S. H. Sackey, J. A. Ansere, J. H. Anajemba, M. Kamal and C. Iwendi,"Energy Efficient Clustering Based Routing Technique in WSN using Brain Storm Optimization," 15th International Conference on Emerging Technologies (ICET), Peshawar, Pakistan, 2019, pp. 1-6, doi: 10.1109/ICET48972.2019.8994740.

[14]R. Singh Kshetrimayum,"Fundamentals of MIMO Wireless Communi- cations", Cambridge University Press, 2017.

[15] V. A. Beulah and S. Markkandan,"Performance Analysis of Precoding Techniques for Massive MUMIMO Systems", IEEE Sponsored 2nd International Conference on Innovations in 
Information Embedded and Communication Systems ICIIECS15, 2015.

[16] E. Perahia and M. X. Gong,'Gigabit wireless LANs: An overview of IEEE 802.11ac and 802.11ad", ACM SIGMOBILE Mobile Computer. Communication Rev., vol. 15, no. 3, pp. 23-33, Jul. 2011.

[17] A. Gupta and R. Kumar Jha,"A Survey of 5G Network: Architecture and Emerging Technologies", IEEE Digital Object Identifier 10.1109/AC- CESS.2015.2461602, July 28, 2015.

[18] C. Shepard, H. Yu, N. Anand, L. E. Li, T. L. Marzetta, R. Yang and

L. Zhong,"Argos: Practical many-antenna base stations," in ACM Int. Conf.Mobile Computing and Networking (MobiCom), Istanbul, Turkey, Aug. 2012.

[19] J. Hoydis, C. Hoek, T. Wild, and S. ten Brink, "Channel measurements for large antenna arrays," in IEEE International Symposium on Wireless Communication Systems (ISWCS), Paris, France, Aug. 2012.

[20] J. Nam, J.-Y. Ahn, A. Adhikary, and G. Caire,'Joint spatial division and multiplexing: Realizing massive MIMO gains with limited channel state information," in 46th Annual Conference on Information Sciences and Systems (CISS), 2012.

[21] K. Jyoti Mohan, O. Gogoi and P. Gogoi, "Interference Cancellation in Massive MIMO Base Stations with Certain Precoding Techniques in Faded Environment", IEEE International Conference on Signal Processing and Integrated Networks, 2014.

[22] L. Pu-Hsuan and T. Shang-Ho, "Performance analysis and algorithm designs for transmit antenna selection in linearly precoded multiuser MIMO systems", IEEE transactions on Vehicular Technology, VOL. 61, NO. 4, pp.1698-1708, 2012.

[23] P. Varshney and H. Jain,'Duality Analysis and Improving the Capacity of Multi-Input MultiOutput Channel using Dirty Paper Coding Tech- nique", IEEE International Conference on Computer, Communication and Control, 2015.

[24] S. H. Sackey, J. H. Anajemba, S. N. Kofie, G. K. Gapko, "An Efficient Data Transmission in Smart Grids Using Edge Computing", International Journal of Progressive Sciences and Technologies, [S.1.], vol. 14, no. 1, p. 8692, Apr. 2019.

[25] Y. Liu, Z. Ding, J. Shi, W. Yang and P. Zhong,'Large System Analysis of Linear Precoding in Massive MIMO Relay Systems," 2018 IEEE 87th Vehicular Technology
Conference (VTC Spring), Porto, 2018, pp. 16, doi: 10.1109/VTCSpring.2018.8417840. 